\title{
DEGUM zeichnet 1. Klinik in Deutschland als „Interdisziplinäres Ultraschall-Zentrum“ aus
}

Für seine hohe Expertise auf dem Gebiet des Ultraschalls zeichnete die Deutsche Gesellschaft für Ultraschall in der Medizin (DEGUM) im August das Uniklinikum Münster als „Interdisziplinäres Ultraschall-Zentrum" aus. In seinen bereits Ultraschall-zertifizierten Abteilungen Anästhesiologie, Pränatalmedizin und $\mathrm{Ge}$ burtshilfe sowie Kardiologie hat die Klinik nun die Weiterbildungskompetenz erreicht und ist damit das 1. DEGUM-zertifizierte Zentrum in Deutschland. Bundesweit sind derzeit 57 medizinische Abteilungen sowie über 4000 Ärzte DEGUMzertifiziert.

Die Sonografie ist das am häufigsten angewandte Bildgebungsverfahren in der Medizin. Umso wichtiger ist eine professionelle und zuverlässige Anwendung dieses Verfahrens. „Die Wertigkeit der Ultraschallbefunde hängt wesentlich von der Erfahrung des Untersuchers ab“, erklärt Professor Dr. med. Dirk Becker, Präsident der DEGUM. „Wir setzen uns daher intensiv dafür ein, dass in Praxen, Kliniken und medizinischen Zentren die Ultraschallanwender hochqualifiziert und auf dem neuesten Stand der Technik und Diagnostik sind.“ Diesen Qualitätsanspruch erfüllt das Uniklinikum Münster (UKM), da es flächendeckend über sehr gut ausgebildetes Personal und moderne Ultraschallgeräte verfügt. Daher erhielt dasUKMals deutschlandweit 1. Klinik die Auszeichnung der DEGUM als Interdisziplinäres UltraschallZentrum. Die offizielle Zertifikatsübergabe fand am 19. August 2015 am Uniklinikum Münster statt. Die DEGUM war vertreten durch ihren Präsidenten Professor Dr. med. Dirk Becker, Vorstandsmitglied Professor Dr. med. Peter Jecker sowie Geschäftsstellenleiterin Marion SchapheerRisse. Sie überreichten die Urkunde feierlich an die Vertreter der aktuell zertifizierten Abteilungen des UKM (Foto).
Das UKM hat sich in den Bereichen Anästhesiologie, Pränatalmedizin und $\mathrm{Ge}$ burtshilfe sowie Kardiologie von der DEGUM prüfen und zertifizieren lassen. Es ist damit gleichzeitig auch Weiterbildungszentrum für die Ultraschall-Ausbildung anderer Ärzte. „Wir freuen uns, dass das UKM sich auf dem Gebiet des Ultraschalls weiter zertifiziert hat und damit die Versorgungsqualität in Deutschland verbessert. Wir hoffen, dass nun weitere Kliniken nachziehen und die Ausbildung auf einem ähnlich hohen Standard wie die Uniklinik Münster interdisziplinär durchführen“, erläutert Becker, Chefarzt Innere Medizin/Gastroenterologie am Krankenhaus Agatharied im bayerischen Hausham.

In den vergangenen 20 Jahren hat sich die Sonografie-Technik enorm weiterentwickelt und verfügt über ein großes Potenzial. „Leider hinkt jedoch die flächendeckende gute Ausbildung und eine hochwertige Geräteausstattung vielfach hinterher“, kritisiert Becker. So sei beispielsweise die Sonografie und KontrastmittelSonografie in der Hand erfahrener Anwender die beste und schonendste Methode in der Früherkennung von Leberkrebs. Habe der Anwender wenig Ultraschall-Erfahrung, sind andere und teurere Untersuchungsmethoden für den Patienten notwendig. „An diesem Beispiel zeigt sich, dass die Versorgung der Patienten leidet, wenn die fachliche Qualifikation und eine hochwertige Technologie nicht ausgeschöpft werden“, bedauert Becker. „Denn eine hochqualifizierte Ultraschalluntersuchung ist komplex und erfordert eine gute Ausbildung. Die Zeche für die anderen, wesentlich teureren Untersuchungen zahlt in diesen Fällen dann stillschweigend die Versichertengemeinschaft." Die DEGUM fördert daher die Fort- und Weiterbildung von Ultraschall-

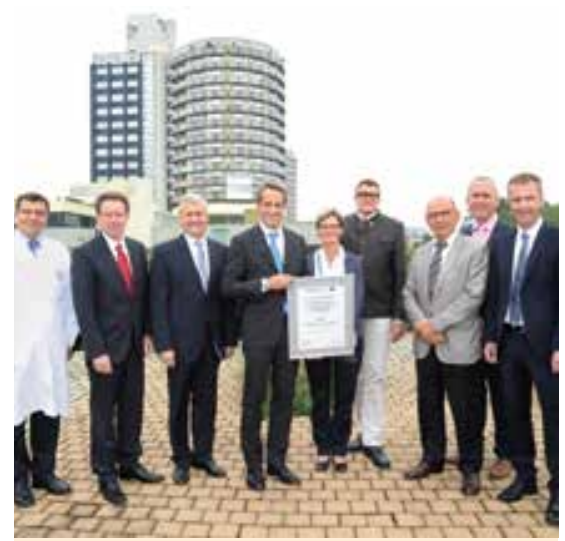

Vertreter der DEGUM (Bildmitte) überreichen die Urkunde am 18. August 2015 offiziell an die Vertreter des UKM. Von links nach rechts: Prof. Dr. med. Johannes Waltenberger (Leiter Department für Kardiologie und Angiologie), Prof. Dr. med. Norbert Roeder (Ärztlicher Direktor UKM), Prof. Dr. med. Jörg Stypmann (Department für Kardiologie und Angiologie, DEGUM Kursleiter [Echokardiografie] Stufe III), Prof. Dr. med. Dirk Becker (DEGUM Präsident), Marion Schapheer-Risse (DEGUM Geschäftsstellenleitung), Prof. Dr. med. Peter Jecker (DEGUM Sekretär), Prof. Dr. med. Dr. h.c. Hugo Van Aken (Direktor Klinik für Anästhesiologie), Dr. med. Thomas Ermert (Klinik für Anästhesiologie, DEGUM Kursleiter [Anästhesiologie] Stufe II), Dr. med. Ralf Schmitz (Klinik für Frauenheilkunde und Geburtshilfe, DEGUM Stufe II [Gynäkologie u. Geburtshilfe]) (Bildquelle: Universitätsklinikum Münster).

anwendern und zeichnet Zentren und Abteilungen aus, die den Qualitätsanforderungen der DEGUM entsprechen. Ärzten bescheinigt die Fachgesellschaft eine entsprechende Qualifikation mit einem Zertifikat der Stufen I bis III. 